Bulletin d'Histoire Contemporaine de I'Espagne

$54 \mid 2020$

Les espaces du politique dans l'Espagne du Trienio liberal (1820-1823)

\title{
Réflexions méthodologiques sur la place du Trienio liberal dans le processus de sortie de l'Ancien Régime
}

Reflexiones metodológicas en torno al lugar del Trienio Liberal en el proceso de salida del Antiguo Régimen

Methodological reflections on the place of the liberal Trienio in the process of leaving the Old Regime

Jean-Philippe Luis ( $($ )

\section{OpenEdition}

Journals

Édition électronique

URL : http://journals.openedition.org/bhce/1211

DOI : 10.4000/bhce.1211

ISSN : 1968-3723

Éditeur

Presses Universitaires de Provence

Édition imprimée

Date de publication : 1 janvier 2020

ISSN : 0987-4135

Référence électronique

Jean-Philippe Luis $(\dagger)$, «Réflexions méthodologiques sur la place du Trienio liberal dans le processus de sortie de l'Ancien Régime », Bulletin d'Histoire Contemporaine de l'Espagne [En ligne], 54 | 2020, mis en ligne le 01 juillet 2020, consulté le 31 décembre 2020. URL : http://journals.openedition.org/bhce/ 1211 ; DOI : https://doi.org/10.4000/bhce.1211

Ce document a été généré automatiquement le 31 décembre 2020.

Bulletin d'histoire contemporaine de l'Espagne 


\title{
Réflexions méthodologiques sur la place du Trienio liberal dans le processus de sortie de l'Ancien Régime
}

\author{
Reflexiones metodológicas en torno al lugar del Trienio Liberal en el proceso de \\ salida del Antiguo Régimen \\ Methodological reflections on the place of the liberal Trienio in the process of \\ leaving the Old Regime
}

Jean-Philippe Luis ( $($ )

\section{NOTE DE L'ÉDITEUR}

C'est avec une énorme tristesse que nous avons appris la disparition de Jean-Philippe Luis qui nous a quitté le 27 octobre 2020. Nous perdons là un grand historien et un homme de bien.

1 Après deux décennies de relatif désintérêt, le Trienio liberal redevient un sujet d'étude porteur, ce qui est probablement lié au bicentenaire de l'avènement de ce régime original dans l'Europe post-napoléonienne, mais aussi à la recherche de nouvelles grilles de lecture des événements révolutionnaires du début du XIX ${ }^{\mathrm{e}}$ siècle. En effet, le renouveau des études sur le libéralisme espagnol ${ }^{1}$, sur la nature de la société d'Ancien Régime ont remis en cause l'interprétation forgée dans les années 1970-1980 par Alberto Gil Novales et Josep Fontana ${ }^{2}$. Au travers d'une synthèse et d'ouvrages généraux sur la révolution libérale qui ont fait longtemps autorité ${ }^{3}$, ces derniers avaient imposé une vision téléologique de l'événement, le présentant, au sein d'un cycle révolutionnaire ouvert par les Cortès de Cadix et se terminant par les moments révolutionnaires de 1835-37, comme une révolution démocratique confisquée par les 
élites libérales, qui aurait pu déboucher sur une démocratie sociale aux accents présocialistes. Pour ces auteurs, la raison de l'échec se situait dans l'alliance d'une bourgeoisie libérale trop faible, contrainte de s'allier avec les forces d'Ancien Régime éclairées, au détriment des aspirations populaires exprimées par la révolution. Fontana affirmait même : "uno de los grandes dramas del movimiento obrero en el siglo XIX fue el de creer en la vocación revolucionaria de la burguesía $»^{4}$.

2 Les travaux menés depuis la fin des années 1980 ont remis progressivement en cause cette interprétation qui, débarrassée du contexte idéologique particulier des années 1970, résiste pourtant encore sur certains points. Éviter toute perspective téléologique impose de se méfier d'une approche qui chercherait forcément dans le Trienio Liberal les premiers pas de la démocratie représentative que nous connaissons ou d'une démocratie sociale telle qu'elle peut être imaginée depuis la deuxième moitié du $\mathrm{XX}^{\mathrm{e}}$ siècle. En effet, les régimes politiques européens du XIX siècle ne doivent pas être perçus comme des longues transitions, mais comme des moments historiques ayant leur propre cohérence. Le Trienio liberal doit être intégré dans la problématique large de la sortie de l'Ancien Régime des sociétés européennes de la fin du XVIII ${ }^{\mathrm{e}}$ siècle au milieu du siècle suivant. Cette sortie est marquée par l'effondrement de la croyance en un monde considéré comme naturel, divin, monarchique et corporatif5 et par "le désinvestissement symbolique et affectif qui transforme la relation à l'autorité, dépouillée de son ancienne transcendance $»^{6}$. Le nouveau monde est caractérisé par la sacralisation de la propriété individuelle, par la fin des privilèges du clergé et de la noblesse et par l'avènement d'une identité collective nouvelle, la nation, une nation souveraine. Le Trienio liberal doit être analysé d'une part à l'aune d'interprétations nouvelles de la sortie de l'Ancien Régime, qui insistent sur les aspects culturels et anthropologiques, économique, sur la relation dialectique entre révolution et contrerévolution, et d'autre part sur de nouveaux travaux empiriques, menés à l'échelle locale, dont le plus dense est la thèse de Jordi Roca Vernet sur Barcelone ${ }^{7}$. Il s'agit aussi de relire l'événement au travers de grilles de lecture théoriques qui ne se limitent pas à celles des années 1970-1980. Sans prétention à l'exhaustivité, le propos est ici de s'appuyer sur de telles grilles pour appréhender trois des thèmes majeurs qui permettent de caractériser l'expérience du Trienio liberal et de tenter de positionner celle-ci dans le processus de sortie de l'Ancien Régime et de construction d'un monde nouveau: la nature de la rupture révolutionnaire, la politisation du corps social, la résistance des conceptions corporatives.

\section{La nature de la rupture révolutionnaire}

3 Nul ne conteste le caractère révolutionnaire, au sens de rupture avec un ordre établi, de l'expérience politique des années 1820-1823. Toutefois, il n'est pas facile de déterminer une espérance commune aux acteurs de cette révolution, ce qui est encore plus vrai si l'on considère le déclenchement de la rupture, en février-mars 1820, avec le soulèvement des villes, en réponse au soulèvement de Las Cabezas de San Juan, le $1^{\mathrm{er}}$ janvier de cette année-là. La situation n'est alors pas celle d'une force réactionnaire prétendant conserver tout ce qui est ancien, face à une force révolutionnaire dotée d'une idéologie cohérente, à l'exception de quelques secteurs politiques très étroits. Pourtant, ces soulèvements sont traditionnellement présentés dans l'historiographie des années 1970 et 1980 comme des révoltes populaires dont la nature révolutionnaire aurait été immédiatement détournée par les élites locales, y compris par celles qui 
s'affirmaient comme libérales. Alberto Gil Novales écrivait ainsi que le mouvement populaire barcelonais " políticamente sale derrotado por la mayor capacidad de maniobra de los que solo quieren que cambien las palabras », et que dans le pays, dès la mi-mars 1820, “la revolución quedó burlada " ${ }^{8}$. La révolution aurait donc été de nature populaire, mais aurait été confisquée dès ses origines.

Derrière ces affirmations, il y a une conception du monde social comme un ensemble de secteurs structurés horizontalement en fonction des modes socio-économiques de domination sociale. La logique était d'origine marxiste, modulée par l'intervention d'éléments culturels issus de l'héritage de Gramsci et de Bourdieu. Or, cette perception est nuancée ou remise en cause par plusieurs travaux issus de l'économie, de la sociologie ou des sciences politiques. A la fin du siècle dernier, les nuances apparurent y compris dans la mouvance bourdieusienne, en particulier sous la plume de Michel Dobry qui, tout en conservant la même logique interprétative globale, insiste sur les moments particuliers que sont les crises politiques majeures. En effet, celles-ci remettent en cause la cohérence des secteurs qui structurent l'espace social (il parle de “désectorisation conjoncturelle de l'espace social» et de crise "en tant que transformations d'état $»^{9}$ ) ce qui conduit à des situations provisoires de " conjonctures fluides" marquées par un brouillage des frontières, des repères et par des actions menées selon des calculs se faisant "selon des voies sensiblement distinctes de celles qui caractérisent les contextes dans lesquels l'individu et le groupe ont à leur disposition des instruments d'anticipation et des points de repère institutionnalisés et familiers $»^{10}$. D'où le caractère conjoncturel et peu prévisible des actions menées, ce qui nous renvoie au propos antérieur de la diversité des horizons d'attente des populations soulevées en mars 1820. La crise n'est alors pas conçue chez Dobry comme une vraie rupture, mais comme une phase de réajustements des secteurs sociaux déjà existants.

D'autres démarches théoriques, fondées sur une autre conception du champ social, remettent en cause la lecture traditionnelle de la rupture de 1820. En simplifiant à l'extrême, et en vue d'un parallèle avec le cas concret du Trienio liberal qui nous intéresse ici, on peut différencier deux types d'interprétations. La première insiste sur une rupture verticale (et non prioritairement en terme de classes) dans le champ des forces sociales. C'est le cas de Douglas North et de sa théorie des "coalitions dominantes » pour qui la crise renvoie à l'épuisement d'un mode de coalition des élites dans le cadre d'un "ordre social fermé", ce qui conduit au retour de la violence inhérente à toute société ayant perdu les institutions et les sources de légitimité qui lui avaient jusqu'alors permis de réguler la violence ${ }^{11}$. La sortie de crise passe alors par la constitution d'une nouvelle coalition dominante. On trouve aussi une cassure du champ social chez Charles Tilly pour qui un processus révolutionnaire est enclenché quand survient " une scission claire et nette dans le corps politique, telle qu'un grand nombre de citoyens ont dû choisir entre des prétentions conflictuelles à leur loyauté et à leur obéissance, et que de nombreux intérêts établis se sont trouvés en butte à des menaces nouvelles $»^{12}$.

6 Un autre courant regroupe des sociologues et des économistes pour qui le champ social ne serait pas constitué de secteurs prédéterminés par les modes socio-économiques de domination sociale, mais serait un espace qui acquerrait du sens par la création sociale de normes, de conventions. Cette capacité à donner du sens viendrait de l'acceptation d'un pouvoir symbolique capable de donner une légitimité aux démarcations créées, ce qui conditionnerait ainsi la lecture que les individus se faisaient du monde social. Ces 
théories que l'on retrouve en particulier dans le courant de l'économie des conventions ${ }^{13}$ s'opposent à la vision marxiste révisée par Bourdieu, mais aussi au modèle libéral walrasien des économistes pour qui l'équilibre social viendrait de la combinaison rationnelle des intérêts des individus. Le courant de l'économie comportementale (en particulier le prix Nobel Richard Thaler), tout comme celui des conventions est convaincu que les hommes sont dotés d'une rationalité limitée et qu'interviennent dans les choix humains de nombreux autres facteurs que l'intérêt, en particulier des facteurs de nature morale ou cognitive.

7 Ainsi, la crise, la rupture quand elle est majeure, viendrait de l'effondrement brutal, au niveau de l'individu comme de collectifs, de la croyance en la légitimité d'un type de relations sociales et de l'ensemble des représentations qui légitime celui-ci. Toutefois, faire face à la crise ne se fait qu'avec des matériaux déjà existants. L'analyse doit donc prendre "en compte les croyances et les valeurs qui peuvent être mobilisées comme justifications pour l'action. Ces croyances et ces valeurs constituent une cosmologie implicite que les individus mobilisent quand ils engagent des échanges et des transactions $»^{14}$. Ceci explique la complexité interprétative de crises comme celles de la guerre d'Indépendance ou du Trienio Liberal qui y est intimement liée, complexité illustrée par la grande plasticité et hybridation du langage des acteurs politiques et sociaux, tout comme celle des notions, des pratiques et des représentations.

S'inspirer de ces théories signifie considérer avant tout l'expérience concrète de l'acteur social, décrypter les arguments moraux employés, les notions mobilisées, chercher le sens des classifications et des institutions créées par ces acteurs, en ayant à l'esprit qu'elles ne sont pas des écrans de fumée masquant des modes de domination socio-économiques.

Jean-Pierre Dedieu a tenté de rapprocher la théorie des conventions de la situation historique concrète de la chute de la monarchie espagnole ${ }^{15}$, c'est à dire d'un monde qui, avant la crise, pensait dans son immense majorité que la monarchie catholique était un horizon naturel et indépassable. La culture juridictionnelle catholique qui servait de ciment à cette société fournit un exemple très parlant de conventions. D'après Dedieu, deux conventions majeures structuraient la société de ce temps : la convention royale et la convention religieuse. La crise correspond à la recherche de conventions alternatives qui mettent forcément un temps long à s'imposer. L'effondrement de l'État monarchique en 1808 et la création de nouvelles options politiques institutionnalisées (le régime de Joseph I, le libéralisme des Cortès de Cadix) ou informelles (la contre-révolution) eurent pour conséquence pour les acteurs de ce temps que le monde dans lequel ils vivaient ne pouvait plus être considéré comme naturel et que l'ordre politique pouvait être bouleversé. Avec la rupture revient l'incertitude face au présent et au futur, un temps doté d'un fort potentiel de violence car le processus n'a pas la même temporalité dans tous les secteurs et tous les territoires de la société. Cela signifie que les individus ne donnent pas forcément le même sens à leurs actions, ce qui apparaît très clairement dans la bataille pour le langage qui caractérise le monde euro-américain lors de la sortie de l'Ancien Régime ${ }^{16}$. Les acteurs en étaient d'ailleurs conscients, ainsi, un rédacteur de la Gaceta de Madrid écrivait dans les premières semaines de la restauration de l'absolutisme en juillet 1823 : “ en lengua masónica, las palabras justicia, probidad y aún conocimiento, no tienen el mismo significado que en la lengua castellana, pues en algunos casos significan todo lo contrario $»^{17}$. 
10 Dans ces moments d'incertitude, l'individu se découvre doté d'un degré d'autonomie beaucoup plus important que lors des phases de stabilité. Pour Mary Davis, les actions des acteurs sociaux ne sont pas prédéterminées, mais dépendent des possibilités et des limitations offertes par le contexte social et par le potentiel relationnel de chacun ${ }^{18}$. Cette sociologie tient ainsi compte de la capacité d'arbitrage des individus entre plusieurs options possibles dans un contexte donné. Concrètement, cela signifie que nous devons nous pencher aussi sur ces moments de crise à partir de cas individuels qui peuvent nous apprendre beaucoup sur les arbitrages et les compromis réalisés par les acteurs sociaux face à des situations inédites. Ces considérations peuvent être reliées aux travaux de Haim Burstin et sur ce qu'il nomme “ le protagonisme ». Dans sa volonté de proposer une nouvelle lecture de la Révolution Française, cet auteur nous décrit ce moment de crise sous la forme d'une “dialectique efficace entre l'individuel et le collectif » qui n'est ainsi ni “ un engrenage inexorable (...) ni même une juxtaposition fortuite d'évènements (...) mais un ensemble où les différents éléments, soumis à la pression d'un certain nombre de circonstances, finissent par s'aligner dans une séquence particulière qui n'est pas pour autant prédéterminée ». Ceci conduit à “donner toute son importance à l'étude des pratiques et des comportements individuels qui doivent être saisis (...) dans leur immédiateté, indépendamment de la manière dont la science politique les élaborera par la suite $»^{19}$.

11 L'apport de cette diversité théorique et épistémologique conduit ainsi à penser la brève expérience révolutionnaire du Trienio liberal comme une étape du moment historique de sortie de l'Ancien Régime qui suit l'effondrement de l'État monarchique en 1808, une étape qui mélange l'ancien et le nouveau dans une tentative désordonnée et plurielle pour construire un ordre nouveau dont le sens n'était ni clair pour les contemporains, ni inscrit dans les faits qui s'y sont déroulés. Ceci permet de comprendre des situations apparemment aussi incohérentes que celles de territoires s'engageant dans la contrerévolution, après avoir refusé de payer les dîmes ${ }^{20}$.

\section{Le Trienio liberal et la notion de politisation}

12 Forgée par les travaux de Maurice Agulhon et des politologues étatsuniens, cette notion n'a été adoptée que relativement tardivement pour l'aire hispanique ${ }^{21}$. Elle a été reprise par François-Xavier Guerra à partir des territoires américains ${ }^{22}$, ce qui s'est avéré aussi particulièrement fécond pour l'analyse de la péninsule ibérique. Depuis le milieu des années 2000 et en particulier à la faveur des travaux liés à la célébration du bicentenaire de la guerre d'Indépendance, cette notion de politisation (on parle aussi parfois de naissance de la politique moderne ${ }^{23}$ ) est largement utilisée et offre d'intéressantes portes d'entrées à l'étude du Trienio liberal.

\section{Les étapes de la politisation}

13 La politisation est le moment où des individus et des communautés se découvrent acteurs potentiels du fonctionnement et/ou de l'organisation des règles et des valeurs qui régissent la dévolution du pouvoir politique et qui légitime les actions de ce dernier. Dans le cas de la sortie de l'Ancien Régime, ce processus s'étend sur un temps plus ou moins long caractérisé par des phases d'accélération (les révolutions, les guerres) et de ralentissement, en sachant qu'une fois ce processus entamé, il n'y a pas de retour en arrière possible. Une forme de consensus s'est aujourd'hui fait pour 
considérer que la politisation antérieure à 1808 était limitée à des groupes d'individus peu nombreux et que la sphère du politique ne s'ouvre brutalement pour la majorité de la population qu'à partir de l'effondrement de l'État monarchique en $1808^{24}$. Le Trienio liberal est indéniablement un moment d'accélération de la politisation dont tous les travaux portant sur le discours autour de la constitution de Cadix et sur la sociabilité libérale témoignent.

Cette réalité n'est cependant pas sans laisser de nombreuses zones d'ombres. Comment les semaines révolutionnaires de février et mars 1820 ont-elles pu avoir lieu, avec la revendication d'un retour à la constitution de Cadix, après six années d'un absolutisme despotique, supprimant toute presse non officielle et ne laissant place à aucun espace de débat? En d'autres termes, les moments entre deux phases révolutionnaires sontelles seulement des parenthèses? Dans le cas qui nous intéresse, la force des soulèvements de la fin de l'hiver 1820 entre en contradiction avec ce qui est le plus souvent affirmé dans l'historiographie, à savoir que les idées libérales ne purent pas imprégner en profondeur la société à cause de la brièveté de l'expérience constitutionnelle des Cortès de Cadix, comme le prouverait la facilité avec laquelle Ferdinand VII et les absolutistes mirent fin au régime constitutionnel en mai 1814.

Pour comprendre ce paradoxe, deux hypothèses se présentent : soit la politisation s'est poursuivie durant le sexenio absolutiste, soit les insurrections de 1820 ne furent pas vraiment libérales. Je crois que les deux facteurs explicatifs peuvent se mêler. En effet, le premier point renvoie aux formes informelles de la politisation, c'est-à-dire, à tout ce qui se développe en dehors des vecteurs puissants et connus de politisation (la presse, les espaces de sociabilités tels que les cafés, les sociétés patriotiques, les cérémonies officielles...). Il s'agit d'une politisation inscrite dans la vie quotidienne, faite de discussions entre amis, de gestes symboliques, de rebellions qui à première vue ne sont pas politiques et qui ont lieu dans des espaces traditionnels de sociabilité comme la rue, le marché, la corporation, ou dans des espaces nouveaux créés à partir du déclenchement du processus révolutionnaire ${ }^{25}$. Nous souffrons dans ce domaine d'un problème de sources et d'études trop peu nombreuses sur le sexenio absolutisme de 1814-1820. Certes, sont connus les pronunciamientos libéraux de Cadix, Valence et de Barcelone où l'action militaire de Lacy a été accompagnée d'une participation des métiers. Mais quels en furent les échos sur le territoire de la monarchie dans une société sans aucune liberté de la presse?

Par ailleurs, il y a dans les soulèvements urbains de 1820 une bonne part de rejet de l'expérience concrète des années de restauration 1814-1820 et de dépit après l'espoir d'un retour à " la normale ", après les six années terribles de la guerre d'Indépendance. Cela signifie qu'il faut probablement remettre en cause l'idée suivant laquelle l'entrée triomphale de Ferdinand VII en 1814 était le signe d'un large attachement à l'Ancien Régime et d'une ignorance des principes du libéralisme. Ce que la foule souhaitait à cette date était avant tout la fin de la guerre, du chaos, de l'incertitude, le retour à l'ordre et non pas forcément le retour à l'absolutisme. Les travaux récents d'Emilio La Parra montrent d'ailleurs que la restauration n'était pas inéluctable, qu'elle n'était qu'une des hypothèses de sortie de crise. Il y avait d'autres options que le roi a su écarter ${ }^{26}$. Le coup de force absolutiste niait l'expérience collective des années de guerre et de révolution et entretenait l'illusion d'une parenthèse qui n'aurait fait que perturber provisoirement une normalité retrouvée ${ }^{27}$. Josep Fontana a d'ailleurs montré avec talent il y a longtemps que la faible résistance à la révolution de 1820 était liée à 
l'impasse idéologique et culturelle dans laquelle se trouvait le pouvoir et à l'augmentation générale des impôts qui avait affecté l'ensemble du corps social, y compris le clergéer ${ }^{28}$. Les soulèvements de 1820 combinaient ainsi d'une part ce dépit et d'autre part les espoirs mis dans les mots de liberté, de rejet du despotisme issus de l'expérience des Cortès de Cadix.

Par ailleurs, ces soulèvements ne doivent pas être l'arbre qui cache la forêt. Dans de nombreux territoires, la politisation libérale n'avait pas atteint le niveau de celle des villes soulevées. L'exemple de Salamanque peut être reproduit ailleurs : l'installation des institutions libérales ne se fit qu'à partir de la publication de l'ordonnance royale du 9 mars 1820 qui informait que le roi avait prêté serment à la Constitution de 1812 et qui appelait tous les pueblos de la monarchie à organiser des élections afin d'élire des alcaldes et conseils municipaux constitutionnels ${ }^{29}$. Dans ces zones, la politisation libérale ne se développe vraiment qu'à partir de la mise en place du régime constitutionnel.

Après la restauration de ce dernier, il est indéniable que la propagande libérale dans le cadre de la milice nationale, des sociétés populaires, de la presse, des tertulias patriotiques, des cérémonies publiques, des sermons des prêtres libéraux, des chaires destinées à l'explication de la constitution, ont permis une diffusion des valeurs libérales. La densité de la carte des sociétés populaires et des bataillons de la milice nationale sur le territoire suffisent à s'en convaincre. C'est toutefois encore le cas catalan qui a été étudié le plus en profondeur ${ }^{30}$ et il reste à réaliser un vrai travail de synthèse de ces différents vecteurs de politisation sur l'ensemble du territoire de la monarchie. La conséquence est que l'apprentissage de la politique s'est fait avant tout à l'échelle locale avec comme acteurs principaux des classes moyennes urbaines " hétérogènes et fermement établies dans leurs sphères locales d'activité ${ }^{31}$. Elle ne se fait donc pas ou peu par une impulsion du pouvoir central. On est très loin du modèle français d'une révolution de la capitale qui rayonne grâce à de solides relais politiques et culturels, pour la plupart antérieurs à la révolution.

Enfin, la politisation est un processus dynamique, et la dernière phase du régime constitutionnel, en 1822-23, constitue une accélération liée en grande partie à la nécessité de procéder à une mobilisation armée afin de lutter contre la guérilla absolutiste, puis contre les troupes françaises. On sait que la guerre contre les troupes napoléoniennes avait été un moment d'apprentissage de la politique, les armes à la main $^{32}$. L'obligation de défendre la constitution a réactivé ce mécanisme et contribué à renforcer l'ancrage des idéaux libéraux dans certains secteurs. Le recrutement de la milice nationale s'est alors nettement étendu en direction des couches populaires, ou plus précisément de l'artisanat, comme à Valence où certains bataillons étaient exclusivement composés d'artisans ${ }^{33}$. Selon un processus bien connu, ce contexte de guerre a contribué localement à une radicalisation, comme à Barcelone lors des journées révolutionnaires du 15 avril et du 4 août $1823^{34}$. On peut affirmer la même chose pour la contre-révolution: des couches sociales peu politisées ont pu se découvrir absolutistes devant les attaques menées par le régime libéral à l'égard des libertés collectives, de l'Église ou devant la maladroite politique fiscale adoptée ${ }^{35}$. Pour les libéraux comme pour les absolutistes, a joué à plein le mécanisme connu suivant lequel l'identification d'un ennemi est l'un des moyens les plus efficaces pour constituer une communauté et un sentiment d'appartenance ${ }^{36}$. 
20 L'avancée rapide des troupes des cent mille fils de Saint Louis en 1823 peut laisser penser que la mobilisation révolutionnaire était peu profonde, et les rares cas étudiés montrent que le ressort patriotique de 1808 ne jouait plus quinze ans plus $\operatorname{tard}^{37}$. Toutefois, il ne faut pas surestimer la facilité avec laquelle les troupes françaises l'ont emporté. D’une part car la résistance de plusieurs villes du littoral méditerranéen, jusqu'en novembre 1823, soit plusieurs semaines après la chute du régime constitutionnel, indique un niveau de mobilisation localement élevé ${ }^{38}$. D'autre part parce que l'intervention française est plus proche de l'appui à l'une des factions d'une guerre civile qu'à une invasion. A cet égard, l'épisode final du Trienio liberal témoigne d'une politisation libérale intense dans les principales villes du littoral méditerranéen, et d'une politisation contre-révolutionnaire dans une bonne partie du reste du territoire.

21 Quelle place accorder au Trienio liberal comme étape du processus global de la politisation? Il est difficile de répondre plus précisément qu'en parlant d'une accélération de ce processus. Durant la década ominosa, l'expérience des années 1820-1823 a plutôt favorisé la politisation contre-révolutionnaire qui s'est épanouie très tôt dans les insurrections et l'agitation du précarlisme, en particulier avec la guerre des agraviados de 1827 puis durant l'année qui a suivi la rupture entre le roi et son frère Don Carlos, en octobre 1832. A cet égard, le Trienio liberal a constitué une expérience préalable qui a compté dans le développement du carlisme. Du côté libéral, à l'exception de rares épisodes comme celui célèbre de Mariana Pineda, on ne sait pas grand-chose de la réalité de la politisation des années 1823-1833, qui ne pouvait qu'être informelle à cause du niveau très élevé de la répression et de la surveillance. Les complots et les opérations se préparaient pour l'essentiel de l'extérieur du territoire et ils n'eurent qu'un écho très limité à l'intérieur, le modèle du pronunciamiento de Riego décrit par Irene Castells (le soulèvement des villes répondant à l'appel au peuple lancé par les auteurs du pronunciamiento) ne parvenant pas à se perpétuer ${ }^{39}$. Quelle fut l'importance de la mémoire et de l'expérience du Trienio liberal dans la période révolutionnaire qui suivit la mort de Ferdinand VII ? La question est bien connue si l'on s'en tient à une histoire institutionnelle et intellectuelle du politique. Les années 1820-1823 sont celles de la division du mouvement libéral, de l'abandon du modèle doceañista de la part de ceux qui souhaitent limiter la participation populaire. Le fil conducteur idéologique entre libéralisme exalté, progressisme et libéralisme démocratique a aussi été étudié ${ }^{40}$. Si l'on se situe du côté des pratiques, des symboles et de leur diffusion, le mode opératoire du soulèvement suivi par l'érection de juntes locales $^{41}$ qui avait été inauguré en 1808 perdure et la constitution de Cadix reste un référent mobilisateur puisque les insurgés de 1835-1836 s'en réclamaient et obtinrent sa restauration provisoire en 1836. Le régime constitutionnel devint aussi un moment pourvoyeur de martyrs de la liberté autour de la figure de Riego ${ }^{42}$. On ignore en revanche le chemin suivi pour perpétuer ces pratiques : quelle est le rôle et l'influence des acteurs des années 1820-23 dans les actions menées lors des journées révolutionnaires des années 1830 ? Ce qui est sûr est que le Trienio liberal fut un moment d'apprentissage de la radicalité libérale par l'expérience, par l'enchaînement des circonstances, la mobilisation permanente du corps social autour des questions politiques, par la menace de la contre-révolution. On peut reprendre à cet égard les propos d'Haïm Burstin : “ On ne naît pas radical ou extrémiste ${ }^{43}$. La mémoire de ce chemin de la radicalité a probablement été réinvestie dans les journées révolutionnaires des années 1830 . Le réinvestissement d'une activité menée dans les 
années 1820-1823 se retrouve aussi dans les demandes de réhabilitation dans un emploi ou dans une décoration qui avait été perdus pendant la décennie absolutiste. Ainsi, nombre d'anciens employés de l'administration mis sur la touche en 1823 retrouvèrent des postes à partir de $1835^{44}$.

\section{Une révolution populaire?} des soulèvements a la radicalité des journées révolutionnaire de 18 Par ailleurs, la première politisation, celle du début de la guerre d'Indépendance s'est faite dans un sens contre-révolutionnaire. En effet, les élites opposées aux Français durent dans un temps très bref mobiliser les couches populaires en trouvant des éléments d'explication à l'effondrement de la monarchie pour justifier la nécessité de la lutte armée. Or, ces éléments simples, immédiatement mobilisables ne pouvaient qu'être issus de la tradition. Par la suite, cette rhétorique contre-révolutionnaire est restée très largement dominante dans les mouvements de résistance aux Français ${ }^{45}$. Cet argument et celui de la déception forte à l'égard des espoirs qui avait été soulevés avec le retour du roi en 1814 permettent de nuancer l'hypothèse d'une radicalité populaire dans les journées de février mars 1820. Il y a bien politisation dans les villes qui se sont soulevées car le peuple est acteur du changement et se considère légitime dans ce rôle. Il y a bien sûr un appel à la restauration de la constitution de Cadix, à la lutte contre le despotisme mais, outre le fait que ce mouvement ne touche que quelques villes, on ne peut en déduire que cette rupture de 1820 soit par essence une révolution populaire, démocratique et sociale.

Ce qui est en revanche très convaincant est l'effort des élites libérales pour diffuser leur idéal politique. L'éducation des masses faisait partie des projets libéraux, dans une démarche paternaliste. Lors d'une session des Cortès du printemps 1822, le député Zulueta réclamait pour les pueblos " fuera del nivel de la verdadera ilustracion del siglo » la création d'écoles de primeras letras, et la rédaction de catéchismes politiques " al alcance de los labradores, para que vayan empapándose en la doctrina constitucional así como en la cristiana ». Il opposait ces derniers aux autres pueblos " más ilustrados y adelantados en la civilización, tales como las grandes capitales y los pueblos litorales, que por las circunstancias de su mayor conversación y trato con toda clase de gentes, sus escuelas o liceos de mejor gusto, $u$ otros accidentes favorables, han tenido más ocasión de desplegar los conocimientos humanos, de 
adquirir nociones de política, de apreciar las ventajas de la libertad y de sentir el temor de su pérdida $»^{46}$.

Cet effort d'éducation politique se faisait au travers de la participation à la milice nationale et à toute une série d'institutions déjà évoquée qui sont autant de vecteurs de la politisation, en particulier les sociétés patriotiques, objet de l'ouvrage majeur d'Alberto Gil Novales ${ }^{47}$. On retrouve ici la place fondamentale des " intermédiaires de la modernité » mis en lumière depuis longtemps par Maurice Agulhon ${ }^{48}$, c'est-à-dire de ces hommes issus des élites ou des classes moyennes urbaines, juristes, employés, qui tentaient de diffuser la connaissance de la constitution et de faire participer les milieux populaires au débat public via des lectures publiques, des discours... ${ }^{49}$. Le rôle d'éducation politique de la milice nationale s'est d'abord concrétisé en direction des couches moyennes, celle des propriétaires, puis, à partir de 1822 vers les franges supérieures des milieux populaires, quand il fallut trouver des hommes prêts à défendre le régime devant les attaques de la contre-révolution.

$\mathrm{Au}$ total, quelle fut l'efficacité de cet effort de politisation libérale des couches populaires? Il est difficile de donner une réponse globale, car là encore, les situations locales sont diverses. Il apparaît toutefois que l'animation du mouvement libéral a été avant tout menée par des individus issus des élites (la plupart des grands leaders libéraux sont issus de familles appartenant à l'hidalguía des villes du royaume ou au milieu du grand commerce) et des classes moyennes urbaines. En zone urbaine, le libéralisme a sans doute mordu aussi dans les couches supérieures des métiers et, dans plusieurs villes du littoral méditerranéen, le libéralisme radical a su, autour des comuneros, mobiliser les couches populaires. En revanche, le républicanisme a toujours été très marginal et porté par une élite intellectuelle sans réelle capacité mobilisatrice ${ }^{50}$.

Les couches moyennes qui assuraient l'essentiel de l'encadrement du mouvement libéral exprimaient avec force un désir de participation politique et par conséquent un rejet des modes traditionnels de représentation qui privilégiaient jusqu'alors les élites traditionnelles. Les employés représentaient un groupe particulièrement actif et numériquement important parmi ces classes moyennes libérales. Après les militaires, ces derniers constituaient le deuxième groupe parmi les membres des sociétés patriotiques étudiées par Alberto Gil Novales. A Salamanque, la moitié des membres de la milice nationale était issue de leurs rangs en $1820^{51}$. Les sources de l'épuration entamée par le pouvoir absolutiste à partir de l'automne 1823 permettent d'affirmer qu'environ le tiers des employés de l'administration centrale, soit près de 3500 personnes, ont joué un rôle actif dans le mouvement libéral en étant membre de la milice nationale ou en ayant accompagné le gouvernement dans sa fuite jusqu'à Séville lors de l'avancée des troupes françaises des cent mille fils de St Louis durant l'été 1823. Les gouvernements libéraux privilégiaient d'ailleurs ce groupe social en leur accordant des hausses de salaire et en respectant jusqu'en 1822 une de leurs principales revendications, à savoir la stabilité de l'emploi, et la fin des mises à pied arbitraires qui s'étaient multipliées durant la première restauration de l'absolutisme ${ }^{52}$.

$\mathrm{Au}$ total, à l'exception des quelques villes du littoral méditerranéen déjà évoquées, on peut mettre en doute l'ampleur de l'attachement des couches populaires au libéralisme. Ce que l'on observe le plus souvent dans ces milieux est au contraire une politisation contre-révolutionnaire. En effet, c'est un phénomène connu dans de vastes territoires ruraux du nord du pays ${ }^{53}$. La politique fiscale du régime constitutionnel qui s'est avérée 
défavorable aux paysans qui avaient des difficultés à commercialiser leur production joua un rôle majeur en ce sens, tout comme les attaques aux principes et solidarités communautaires. Il semble bien que les couches populaires urbaines furent aussi des foyers de la contre-révolution. La grande violence désordonnée qui accompagna la chute du régime constitutionnel vient pour l'essentiel de ces groupes sociaux. Cette question reste largement à approfondir, mais la réalité et l'ampleur du phénomène ne fait guère de doute. A Séville, le 13 juin 1823, quelques heures après la fuite du gouvernement constitutionnel, "grupos de los barrios populares de Triana, Humeros, San Roque y la Macarena " saccagèrent les habitations des libéraux ainsi que les locaux de la société patriotique, massacrant au passage plusieurs personnes ${ }^{54}$. Les travaux récents d'Álvaro París permettent de repérer de tels phénomènes à Madrid et dans plusieurs autres villes ${ }^{55}$. On y trouve très souvent des actes iconoclastes, des persécutions de libéraux et des velléités de soulèvement contre les troupes françaises accusées de protéger les libéraux. Dans la capitale, la tentative de Bessières pour faire entrer ses troupes dans la ville avant l'entrée programmée du duc d'Angoulême pour le 24 mai 1823 fut accompagnée par un soulèvement des quartiers populaires qui voulaient se venger des negros. Le massacre perpétré par les troupes libérales qui étaient encore présentes dans la ville donne une idée de la sociologie de ce soulèvement: "el populacho", "aguadores, bolleros, vivanderas", des femmes, des enfants. Il est fort probable que l'hypothèse présentée par Álvaro París suivant laquelle les arrestations massives de libéraux actifs ou identifiés comme tels, opérées un peu partout par les autorités aient été aussi un moyen de juguler la violence populaire et limiter les massacres.

\section{Conceptions organiques et révolution}

30 La vieille monarchie juridictionnelle de l'Ancien Régime reposait sur une conception organique de la société, marquée par le pluralisme juridique des corps constituant celle-ci. Les travaux sur la constitution de Cadix ont montré la permanence de nombreux éléments s'inscrivant dans une anthropologie catholique et corporative de la société ${ }^{56}$. Ce texte ne se proposait pas d'édifier un État monarchique nouveau fondé sur le respect des droits des individus, mais bien de reconstruire la monarchie sous la forme d'une nation catholique, en constitutionnalisant un ordre social préconstitutionnel. Ceci permet de mieux comprendre l'importance de la politisation à l'échelle locale car le municipio était encore pensé au début du XIX ${ }^{\mathrm{e}}$ siècle comme un corps largement autonome qui fut capable de se proclamer dépositaire de la souveraineté royale entre 1808 et 1810 et de s'incarner sous la forme de juntes, jusqu'à ce que le décret du 24 septembre 1810 ne proclame la souveraineté de la nation ${ }^{57}$. Cette conception locale et pluriséculaire de la souveraineté ne s'est pas évaporée brutalement. Elle reste un ressort essentiel de la légitimité des soulèvements de 1820 .

31 Le libéralisme qui se dessine ainsi est d'essence profondément religieuse. L'accompagnement religieux de tout acte politique contribue aussi à le prouver. Il suffit pour s'en convaincre de relire les articles de la constitution qui détaillent avec une grande précision les modalités d'élection des députés, en commençant à l'échelle de la paroisse (présence du curé de la paroisse, messe solennelle de Espíritu santo, Te deum... : art. 46, 47, 48 et 58 de la constitution). Avant même cette relecture de la constitution, dès les années 1980, les travaux de Gérard Dufour et d'Emilio La Parra avaient révélé l'existence d'un clergé libéral très actif à l'époque du Trienio liberal. Les sermons 
libéraux publiés et commentés par Gérard Dufour offrent un exemple passionnant de lecture de la révolution et de la constitution sous l'angle des saintes écritures ${ }^{58}$. A l'échelle de l'Europe, plusieurs travaux récents insistent sur le caractère profondément religieux du libéralisme dans le monde catholique ${ }^{59}$. Même de manière implicite, la religion reste la justification ultime du pouvoir et la grille de lecture évidente du présent pour l'immense majorité des acteurs politiques, libéraux ou absolutistes, durant le Trienio liberal. Cette réalité peut même être étendue à la première moitié du XIXe siècle. Absolutistes comme libéraux partageaient en particulier un même attachement à l'unité de la communauté, une communauté politique pensée et assimilée à la communauté catholique. La conséquence en est l'exclusion des opposants politiques de la communauté, si nécessaire par l'élimination physique. Les textes contre-révolutionnaires sont nombreux en la matière, et font très souvent le parallèle avec la lutte contre les hérésies ${ }^{60}$, mais on retrouve la même logique chez les libéraux.

Certes, on sait que le Trienio liberal a connu les premières manifestations anticléricales violentes en Espagne. Toutefois, il ne s'agissait pas de s'attaquer à la religion et encore moins de manifester une volonté déchristianisatrice sur le modèle de la France de la Convention. L'objectif était de lutter contre le clergé contre-révolutionnaire accusé, à juste titre, d'œuvrer contre la révolution ${ }^{61}$. Là encore, la perspective téléologique est à éviter: les massacres d'ecclésiastiques de 1936 ne sont pas de même nature que les exécutions de prêtres qui eurent lieu en 1822 et 1823.

Le catholicisme est le fond anthropologique des conceptions organiques de la société. De manière plus concrète, la prévalence de la communauté sur l'individu et de l'autonomie de celle-ci se retrouve dans plusieurs domaines. J'ai pu le constater dans le monde des employés, pourtant, comme nous l'avons déjà souligné, très marqué par le libéralisme. Parmi ces derniers, le rejet de l'absolutisme est fortement lié à la dénonciation des mises à pied pour raisons politiques qui avaient eu lieu entre 1814 et 1820. L'argument est clair : l'emploi public est considéré comme une propriété dont le détenteur ne pouvait être dessaisi qu'après un procès fondé sur les normes, règlements et pratiques qui régulaient les corps structurant ces emplois. En prononçant des mises à pied arbitraires, la restauration de 1814 avait violé la tradition et l'intégrité de ces corps $^{62}$.

On retrouve ces conceptions organiques dans les milieux populaires. En effet, plusieurs éléments montrent que la mobilisation en faveur de la liberté prend bien souvent tout son sens dans le cadre de la communauté locale. Dans cet imaginaire, toute intromission de l'appareil administratif central était perçu comme une attaque à la liberté de la communauté, à sa capacité d'autogestion, la finalité du pouvoir politique restant celle de rendre la justice et non d'élaborer des normes, des lois applicables à l'ensemble des citoyens sans distinction. Pour ces couches populaires, la politique consistait à "dar a cada uno lo que en derecho le corresponde a partir de actos deliberativos que exigían escuchar a partes en conflicto ${ }^{63}$. Cette perception du monde pouvait expliquer l'attachement de certains secteurs populaires urbains à un libéralisme radical qui puisait dans une lecture organiciste de la constitution de Cadix lecture qu'ils considéraient comme "pure »- leur rejet du libéralisme modéré. Le plus souvent, le même mécanisme expliquait la mobilisation contre-révolutionnaire, y compris lors de la deuxième restauration de l'absolutisme, quand le pouvoir voulut imposer une police d'État indépendante des dispositifs traditionnels de surveillance publique $^{64}$. 


\section{Conclusion}

Lublic de la révolution française peut-être caractérisé comme un espace politique moderne créé dans un environnement culturel et mental largement traditionnel $»^{65}$ écrivait Roger Chartier. Cette affirmation peut s'appliquer de manière encore plus pertinente pour l'Espagne du Trienio liberal. Le maintien des conceptions corporatives dans le mouvement libéral, en particulier dans les catégories moyennes et populaires, entrait en conflit avec la théorie du gouvernement représentatif qui progressait alors fortement dans les secteurs du libéralisme de gouvernement et dans laquelle "el ejercicio de la soberanía no reside en la nación, sino en las personas a quienes la nación ha delegado $»^{66}$. Il s'agit là de deux conceptions de la souveraineté en concurrence. La première dominait dans le libéralisme révolutionnaire et avec elle le discours sur la citoyenneté aux accents démocratiques. Il y a donc un certain anachronisme à assimiler ce courant aux premiers pas d'une démocratie sociale du XXe siècle. L'imaginaire social des acteurs du Trienio liberal restait largement religieux et corporatif, mais dans un contexte nouveau qui avait vu s'effondrer depuis peu le monde de la monarchie traditionnelle que l'on pensait naturel, sans que ces certitudes ne soient remplacées par un avenir clair.

Par ailleurs, le caractère populaire de la révolution du Trienio liberal doit être nuancé. “Who is below? " On peut reprendre ici la question posée par Simona Cerruti dans un article où elle invitait les historiens à la prudence à l'heure de mobiliser la notion d'économie morale d'Edward P. Thompson pour expliquer les révoltes populaires ${ }^{67}$. Si la présence populaire est attestée dans les villes du littoral méditerranéen, les forces vives de la révolution libérale semblent plutôt se situer parmi une fraction des élites et surtout parmi les couches moyennes ainsi que dans la partie supérieure des métiers, ce qui invite à mettre en valeur le potentiel révolutionnaire des classes moyennes dans les phénomènes révolutionnaires de la transition de l'Ancien Régime vers le libéralisme.

En dernier lieu, on ne peut soutenir comme le prétend l'interprétation forgée dans les années 1970-80, que la nature profonde de la rupture politique de 1820 et de l'expérience révolutionnaire ait été celle des secteurs radicaux du libéralisme. Tout d'abord, une telle vision pose un problème méthodologique : on ne peut expliquer 1820 et 1821 par la radicalisation de 1822 et 1823 . On sait que ces phénomènes de radicalisation en contexte révolutionnaire sont liés à un climat d'instabilité permanente combinée à un surinvestissement patriotique de certains acteurs qui ne peut être canalisé par des institutions et enfin à une exacerbation de questions sociales qui, dans cet environnement particulier où tout devient politique, ne parviennent à trouver de réponses. Haim Burstin parle "d'une sorte d'hyperoxigénation de la vie publique " à propos de la Révolution Française ${ }^{68}$. Par ailleurs, le libéralisme est un mouvement pluriel, un "état d'opinion publique", qui ne peut être réduit aux exaltados, ces derniers constituant eux-mêmes une nébuleuse peu cohérente ${ }^{69}$, encore moins aux plus radicaux d'entre eux. Enfin, on ne peut dénier tout caractère révolutionnaire aux secteurs du libéralisme qui n'étaient pas radicaux si l'on prend le sens de révolution comme un changement rapide et profond du système politique et social en place. Les libéraux, quels qu'ils soient, avaient en commun la volonté de refonder la monarchie, de mettre un terme aux privilèges qui étaient attachés à la monarchie traditionnelle, dans le cadre d'une nouvelle communauté souveraine, la nation. Cet objectif est une révolution qui a débuté pendant la guerre d'Indépendance

Bulletin d'Histoire Contemporaine de l'Espagne, 54 | 2020 
et qui s'est bien poursuivi pendant le Trienio Liberal en se concrétisant par l'émergence de nouvelles institutions, de nouvelles élites, par une accélération de la mutation de la structure foncière avec la reprise de la desamortización, par la suppression de l'Inquisition... Sans être forcément radicaux, des hommes et des femmes se sont exilés en 1823 , ou sont morts pour cette cause $\mathrm{e}^{70}$. Pour toutes ces raisons, la thèse du Trienio Liberal assimilé à une révolution populaire, démocratique et sociale confisquée ne rend pas compte de la complexité de ce moment historique. Elle ne peut plus être soutenue, mais peut être analysée comme un objet d'histoire, ramené au rang de mythe propre au contexte historiographique, politique et culturel des années 1970.

\section{NOTES}

1. Parmi de nombreuses publications, voir les deux premiers volumes récents de la Historia de las culturas políticas en España y América latina. Miguel Ángel Cabrera, Juan Pro (coords.), La creación de las culturas políticas modernas 1808-1833, Zaragoza/Madrid, PUZ/Marcial Pons ed., 2014. María Cruz Romeo, María Sierra (coord.), La España liberal 1833-1874, Zaragoza/Madrid, PUZ/Marcial Pons ed., 2014. Voir aussi Jean-Philippe Luis, «Guerre d'Indépendance et libéralisme : à la croisée de deux rénovations historiographiques", dans J. P. Luis (dir.), La guerre d'Indépendance espagnole et le libéralisme au XIX ${ }^{e}$ siècle, Madrid, Casa de Velázquez n 118, 2011, p. 1-14. Jesús Millán, «La primera fase del Estado-nación en España, 1808-1880: cambios sociales y espacios políticos», Cahiers de civilisation espagnole contemporaine, $\mathrm{n}^{\circ} 20,2018$, http://journals.openedition.org/ccec/7259

2. Jesús Millán, «La formación de la España contemporánea: el agotamiento explicativo del “fracaso" liberal », Ayer, n 98, 2015, pp. 243-256. Dans le même numéro se trouve la réponse de Josep Fontana (p. 257-260).

3. Alberto Gil Novales, El Trienio Liberal, Madrid, Siglo XXI, 1980. Josep Fontana, La crisis del Antiguo régimen (1808-1833), Barcelona, Crítica, 1979.

4. Josep Fontana, De en medio del tiempo. La segunda restauración española (1823-1833), Barcelona, Crítica, 2006, p. 308.

5. Jean-Philippe Luis, «La représentation antirévolutionnaire du monde: le cas espagnol (1808-1833) », Siècles, n 43, 2016, URL : http://journals.openedition.org/siecles/3066

6. Roger Chartier, Les origines intellectuelles de la Révolution française, Paris, Le Seuil, 1990, p. 289.

7. Jordi Roca Vernet, La Barcelona revolucionaria i liberal: exaltats, milicians $i$ conspiradors, Barcelona, Fundación Noguera, 2011. Parmi les thèses récentes voir aussi Claudio Calles Hernández, La milicia nacional en Salamanca durante el Trienio liberal (1820-1823), tesis doctoral, Universidad de Salamanca, 2015 ; Sophie Bustos, La Nación no es patrimonio de nadie. El liberalismo exaltado en el Madrid del Trienio Liberal (1820-1823): Cortes, gobierno y opinión pública, tesis doctoral, Universidad Autónoma de Madrid, 2017.

8. Emiliano Fernández del Pinedo, Alberto Gil Novales, Albert Dérozier, Centralismo, ilustración y agonía del Antiguo Régimen (1715-1833), Madrid, Labor, 1992, p. 290-291.

9. Michel Dobry, Sociologie des crises politiques, Paris, Presses de la Fondation nationale des sciences politiques, 1992, p. 126 et 34 .

10. Idem, p. 318. 
11. Douglas North, John Joseph Wallis, Barry R. Weingast, Violence et ordres sociaux, Paris, Gallimard, 2010.

12. Charles Tilly, Les révolutions européennes 1492-1992, Paris, Le Seuil, 1993, p. 368.

13. Rainer Diaz-Bone, Laurent Thévenot, «La sociologie des conventions. La théorie des conventions, élément central des nouvelles sciences sociales françaises ", Trivium, $\mathrm{n}^{\circ} 5$, 2010. URL : http://trivium.revues.org/3626. Philippe Batifoulier (ed.), Théorie des conventions, Paris, economica, 2001.

14. Marcel Calvez, «L'analyse culturelle de Mary Douglas: une contribution à la sociologie des institutions ", SociologieS, Théories et recherches, §.6, URL: http:// sociologies.revues.org/522

15. Jean-Pierre Dedieu, Après le Roi. Essai sur la chute de la monarchie espagnole, Madrid, Casa de Velázquez, 2010.

16. Javier Fernández Sebastián, «Política antigua - política moderna, Una perspectiva históricoconceptual», Mélanges de la Casa de Velázquez, n 34 (2), 2005, p. 165-181.

17. Gaceta de Madrid, 22 de Julio de 1823, p. 157.

18. M. Calvez, « L'analyse culturelle de Mary Douglas... », op. cit., § 8.

19. Haim Burstin, Révolutionnaires. Pour une anthropologie politique de la Révolution Française, Paris, Vendémiaire, 2013, p. 414-415.

20. Jesús Millán, «La herencia política de la revolución liberal », dans La politisation des campagnes au XIX ${ }^{e}$ siècle. France, Italie, Espagne, Portugal, Rome, École française de Rome, 2000, p. 259-286.

21. Jordi Canal, "Maurice Agulhon: historia y compromiso republicano", Historia Social, n² 29 (1997), p. 47-72.

22. Voir l'article fondateur: François-Xavier Guerra, «Pour une nouvelle histoire politique : acteurs sociaux et acteurs politiques ", dans Structures et cultures des sociétés ibéro-américaines, audelà du modèle socio-économique, Paris, CNRS-MPI, 1990, p. 245-260

23. Jean-Philippe Luis, "Considérations pour une histoire de la naissance de la modernité politique en Espagne ", La naissance de la politique moderne en Espagne (milieu XVIII ${ }^{e}$-milieu XIX siècle), Mélanges de la Casa de Velázquez, nº 34 (2), 2005, p. 15-26. Un développement de la réflexion dans "Cuestiones sobre el origen de la modernidad política en España ", Revista de Historia Jerónimo Zurita, n 84, 2009, p. 247-278.

24. Pedro Rújula, «La densificación del universo político popular durante la Guerra de la Independencia », dans Pedro Rújula y Jordi Canal (eds.), Guerra de ideas. Política y cultura en la España de la Guerra de la Independencia, Madrid, Marcial Pons ed., 2012, p. 173-190.

25. Je renvoie au colloque $L a$ "modernisation" à l'épreuve des pratiques. Les espaces alternatifs du politique (Europe-Amérique, XVIII ${ }^{e}$-XIX ${ }^{e}$ siècle), qui s'est tenu à la Casa de Velázquez les 25 et 26 février 2016.

26. Emilio La Parra, Fernando VII. Un rey deseado y detestado, Madrid, Tusquets ed., 2018.

27. Pedro Rújula, «El mito contrarrevolucionario de la "Restauración" ", Pasado y Memoria, Revista de Historia contemporánea, $\mathrm{n}^{\circ}$ 13, 2014, p. 79-94.

28. Josep Fontana, La quiebra de la monarquía absoluta 1814-1820, Barcelona, Ariel, 1971. JeanPhilippe Luis, «La realidad es tozuda. Las contradicciones del absolutismo restaurado frente a la cuestión de la Hacienda ", dans Pedro Rújula, Javier Solans (dir.), El desafío de la revolución. Reaccionarios, antiliberales y contrarrevolucionarios (siglo XVIII y XIX), Albolote, ed. Comares, 2017, p. 49-63.

29. Claudio Calles Hernández, "La revolución de 1820 en Salamanca », Revista de Estudios, n 46, 2001, p. 69-114. 
30. Outre la thèse de Jordi Roca Vernet déjà citée, Ramón Arnabat Mata, «La divulgación popular de la cultura liberal durante el Trienio (Cataluña 1820-1823) », Trienio. Ilustración y Liberalismo, $\mathrm{n}^{\circ}$ 41, 2003, p. 55-83.

31. Isabel Burdiel, María Cruz Romeo, «Old and new liberalism: the making of the liberal revolution, 1808-1844», Journal of Iberian Studies, LXXV, 2000, p. 65-80, p. 67.

32. Pedro Rújula, «La guerra como aprentizaje político. De la Guerra de la Independencia a las guerras carlistas ", dans El carlismo en su tiempo: geografías de la contrarrevolución. I jornadas de estudio del carlismo. 18-21 septiembre 2007. Estella-Pamplona, Gobierno de Navarra, 2008, p. 41-64.

33. María Cruz Romeo, «La sombra del pasado y la expectativa de futuro: jacobinos, radicales y republicanos en la revolución liberal », dans Lluis Roura, Irene Castells (eds.), Revolución y democracia. El jacobinismo europeo, Madrid, Ed. del Orto, 1995, p. 107-138, p. 120.

34. J. Roca Vernet, La Barcelona revolucionaria... op. cit., p. 261-290.

35. Emilio La Parra, Los cien Mil hijos de San Luis. El ocaso del primer impulso liberal en España, Madrid, Síntesis, p. 119-182.

36. Jean-Claude Caron, «Introduction », dossier «L'identification de l'ennemi », Siècles, $\mathrm{n}^{\circ} 31$, 2010, URL : http://journals.openedition.org/siecles/169

37. Gonzalo Butrón Prida, «El mito de 1808. La apelación a la nación española durante la intervención francesa de 1823 ", dans José Antonio Armillas Vicente (coord.), La Guerra de la Independencia. Estudios, Zaragoza, Institución « Fernando el Católico », 2001, p. 1189-1204.

38. Carthagène, défendue par Torrijos, est la dernière ville à se rendre le 30 novembre.

39. Irene Castells Oliván, La utopía insurreccional del liberalismo. Torrijos y las conspiraciones liberales de la década ominosa, Barcelona, Crítica, 1989.

40. María Cruz Romeo, "Los mundos posibles del liberalismo progresista », dans Emilio La Parra, Germán Ramírez (eds.), El primer liberalismo en España, una perspectiva comparada, Valencia, Biblioteca valenciana, 2003, p. 287-314. Jordi Roca Vernet, «Las sociedades patrióticas del liberalismo exaltado al liberalismo democrático (1820-1854): una práctica de sociabilidad formal liberal ", dans Ramon Arnabat i Mata, Montserrat Duch Plana (coord.), Historia de la sociabilidad contemporánea: del asociacionismo a las redes sociales, Valencia, Universidad de Valencia, 2014, p. 39-67.

41. Antoni Moliner i Prada, Revolución burguesa y movimiento juntero en España, Lleida, Milenio, 1997.

42. Victor Sánchez Martín, Rafael del Riego. Símbolo de la Revolución Liberal, Tesis doctoral, Universitat de Alicante, 2016.

43. H. Burstin, Révolutionnaires...op. cit., p. 396

44. Jean-Philippe Luis, «Les paradoxes de la politisation dans la fonction publique ", Mélanges de la Casa de Velázquez, n³4, 2005, p. 143-164, p. 155.

45. Pedro Rújula, «Realismo y contrarrevolución en la Guerra de la Independencia », Ayer, $\mathrm{n}^{\circ}$ 86, 2012, pp. 45-66.

46. Session du 15 juin 1822. Cité dans Sophie Bustos, La Nación no es patrimonio de nadie..., op. cit., p. 147 et 151 .

47. Alberto Gil Novales, Las sociedades patrióticas (1820-1823), Las libertades de expresión y de reunión en el origen de los partidos políticos, Madrid, 1975.

48. Maurice Agulhon, "Monsieur Homais ou le militantisme" dans Histoire vagabonde III. La politique en France d'hier à aujourd'hui, Paris, Gallimard, 1996, p. 43-60.

49. Toute une série d'exemples très précis pour le cas barcelonais dans J. Roca Vernet, La Barcelona revolucionaria..., op. cit. 
50. Jordi Roca Vernet, « ¿Hubo republicanos en el Trienio liberal ? Historia, moral y federalismo en el discurso republicano del primer liberalismo », Revista de Estudios Políticos, $\mathrm{n}^{\circ}$ 156, Madrid, abril-junio (2012), p. 85-123.

51. C. Calles Hernández, La milicia nacional en Salamanca..., op. cit., p. 122.

52. Jean-Philippe Luis, «Libéralisme et fonction publique: l'exemple du Trienio Liberal 1820-1823) », Trienio. Ilustración y Liberalismo, n³0, 1997, p. 47-66 ;

53. Parmi les travaux marquants. Ramón Arnabat i Mata, Revolució i Contrarevolució a Catalunya durant el Trienni Liberal (1820-1823), Tesis doctoral, Barcelona, Universitat Pompeu Fabra, 1999. Du même auteur, « Radiografía de la contrarevolució i l'antirevolució durant el trienni liberal a Catalunya (1820-1823) », Millars: Espai i historia, $\mathrm{n}^{\circ} 23,2000$, p. 77-98. Pedro Rújula, Constitución o muerte. El Trienio Liberal y los levantamientos realistas en Aragón (1820-1823), Zaragoza, ed. de l'Astrale, 2000. Ricardo Robledo Hernández, « Realismo sin carlismo: Notas sobre la Revolución Liberal en tierras de Zamora (1820-1833) », Trienio. Ilustración y Liberalismo, n 50, 2007, p. 95-124. 54. J. Fontana, De en medio del tiempo, op. cit., p. 52-53.

55. Álvaro París, « El fin del Trienio: contrarrevolución popular y terror blanco en 1823 ", Ayer (à paraître). Je remercie Álvaro París de m'avoir donné connaissance de son texte.

56. Dans une bibliographie importante: José María Portillo Valdès, Revolución de nación, orígenes de la cultura constitucional en España, 1780-1812, Madrid, CEPC, 2000 ; José Álvarez Junco, Javier Moreno Luzón (éd.), La Constitución de Cádiz : historiografía y conmemoración. Homenaje a Francisco Tomás y Valiente, Madrid, CEPC, 2006; Marta Lorente (coord.), La Constitución de 1812: miradas y perspectivas. Teoría y Derecho, Revista de pensamiento jurídico, $\mathrm{n}^{\circ} 10,2011$, p. 7-129. Ignacio Fernández Sarasola, La Constitución de Cádiz. Origen, contenido y proyección internacional, Madrid, CEPC, 2011.

57. Marta Lorente, José María Portillo, El momento gaditano. La constitución en el orbe hispánico (1808-1826), Madrid, Congreso de los diputados, 2012.

58. Gérard Dufour, Sermones revolucionarios del Trienio Liberal (1820-1823), Alicante, Instituto de cultura Juan Gil Albert, 1991. Emilio La Parra, El primer liberalismo y la Iglesia. Las Cortes de Cádiz, Alicante, Instituto de estudios Juan Gil-Albert, 1985.

59. Maurizio Isabella, «Citoyens ou fidèles? Religion et révolutions libérales dans l'Europe méridionale des années 1820 ", dans Jean-Claude Caron, Jean-Philippe Luis (eds.), Rien appris, rien oublié? Les restaurations dans l'Europe postnapolonienne (1814-1830), Rennes, Presses Universitaires de Rennes, p. 257-270. Jesús Millán, María Cruz Romeo, «La nación católica en el liberalismo. Las perspectivas sobre la unidad religiosa en la España liberal », Historia y Política, n 34, 2015, p. 183-209. Jesús Millán y María Cruz Romeo, «Iglesia y religión en el liberalismo anterior a la sociedad de masas ", dans Salvador Calatayud, Jesús Millán y María Cruz Romeo (eds.), El Estado desde la sociedad. Espacios de poder en la España del siglo XIX, Alicante, Universitat d'Alacant, 2016, p. $149-186$

60. Jean-Philippe Luis, «La construcción inacabada de una cultura política realista », dans Miguel Ángel Cabrera, Juan Pro (coords.), La creación de las culturas políticas modernas 1808-1833. Historia de las culturas políticas en España y América Latina, t. 1, Zaragoza/Madrid, PUZ/Marcial Pons ed., 2014, p. 319-346.

61. Juan Sisinio Pérez Garzón, « Curas y liberales en la revolución burguesa », Ayer, $\mathrm{n}^{\circ} 27$, 1997, p. 67-100. Emilio La Parra, «Los inicios del anticlericalismo español 
contemporáneo », dans Emilio La Parra, Manuel Suárez Cortina (eds.), El anticlericalismo español contemporáneo, Madrid, Biblioteca Nueva, 1998, p. 17-68.

62. Jean-Philippe Luis, « Les paradoxes de la politisation dans la fonction publique », op. cit.

63. Jesús Izquierdo, «La política como controversia : crisis constitucional y respuesta subalterna en los albores del liberalismo ", dans Miguel Ángel Cabrera, Juan Pro (coords.), La creación de las culturas políticas modernas 1808-1833..., op. cit., p. 251-270, p. 268.

64. Álvaro París Martín, Se susurra en los barrios bajos: policía, opinión y política popular en Madrid, 1825-1827, Tesis doctoral, Universidad Autónoma de Madrid, 2016.

65. Roger Chartier, Les origines intellectuelles de la Révolution française, Paris, Le Seuil, 1990, p. 238.

66. Cité dans Javier Fernández Sebastián, « Democracia », dans Javier Fernández Sebastián, Juan Francisco Fuentes (dirs.), Diccionario político y social del siglo XIX español, Madrid, Alianza editorial, 2002, p. 216-228, p. 218

67. Simona Cerruti, « Who is below ? E. P. Thompson, historien des sociétés modernes : une relecture », octobre-décembre 2015, n4, p. 931-955.

68. H. Burstin, Révolutionnaires...op. cit., p. 295-411.

69. Irene Castells, «Le jacobinisme et le romantisme politique dans la presse du premier libéralisme espagnol (1808-1823)", dans Hommage à Jean-Paul Bertaud: la plume et le sabre, Paris, Publications de la Sorbonne, 2002, p. 173-183.

70. J. Millán, « La formación de la España contemporánea... », op. cit.

\section{RÉSUMÉS}

Le Trienio liberal a été pendant longtemps interprété comme une expérience révolutionnaire, émanation d'un mouvement populaire démocratique. Son échec a même été présenté comme le symbole de l'échec de la révolution libérale. En s'appuyant sur les théories développées depuis la fin du XXe siècle en sciences sociales, en rejetant toute perspective téléologique, cet article tente de montrer que le Trienio Liberal ne peut être réduit à une révolution populaire démocratique confisquée. Cette expérience historique se fait dans un environnement socio-culturel qui reste largement marqué par les conceptions corporatives, dans un territoire où la révolution prit des visages très différents et où les couches populaires furent davantage impliquées dans la contrerévolution que dans une révolution dont le moteur social semble bien les classes moyennes urbaines.

El Trienio Liberal ha sido interpretado durante mucho tiempo como una experiencia revolucionaria, emanación de un movimiento popular democrático. A partir de las teorías desarrolladas desde el final del siglo XX en ciencias sociales y con un planteamiento marcado por el rechazo de las perspectivas teleológicas, este artículo quiere demostrar que el Trienio liberal no puede ser reducido a una revolución popular democrática confiscada. Aquella experiencia histórica tuvo lugar en un universo socio-cultural que seguía muy marcado por las concepciones corporativas, en un territorio donde la revolución aparece como muy diversa y donde las capas populares eran globalmente más implicadas en la contra-revolución que en una revolución marcada en realidad por el papel central de la clase media urbana. 
The liberal Trienio has for a long time been interpreted as a revolutionary experience, emanating from a democratic popular movement. Its failure was even presented as a symbol of the failure of the liberal revolution. Relying on the theories developed since the end of the 20th century in social sciences, rejecting any teleological perspective, this article tries to show that the Trienio Liberal cannot be reduced to a confiscated democratic popular revolution. This historical experience takes place in a socio-cultural environment that remains largely marked by corporate conceptions, in a territory where the revolution took on very different faces and where the popular strata were more involved in the counter-revolution than in a revolution whose social driving seems to be the urban middle classes.

INDEX

Palabras claves : Trienio liberal, liberalismo, clases populares, clase media, 1820-1823, teorías de la revolución

Mots-clés : Trienio liberal, libéralisme, classes populaires, classes moyennes, 1820-1823, théories de la révolution

Keywords : Trienio liberal, liberalism, working class, middle class, 1820-1823, theories of revolution

\section{AUTEUR}

JEAN-PHILIPPE LUIS (

CHEC/MSH, Université Clermont-Auvergne, CNRS, CHEC, F-63000 Clermont-Ferrand, France 